\title{
Efeitos da Composição Domiciliar e da Escala Equivalente sobre as Medidas de Desigualdade de Renda e de Pobreza no Brasil
}

\section{Effects of Household Composition and Scale Equivalent on Measures of Income Inequality and Poverty in Brazil}

\author{
Erik Alencar de Figueiredo**
}

Jorge Luiz Mariano***

\begin{abstract}
Resumo: Este trabalho tem o objetivo de mensurar a sensibilidade de algumas medidas de desigualdade de renda e de pobreza, no Brasil, as variações nas escalas equivalentes e na composição dos domicílios. O estudo segue o referencial teórico desenvolvido por Coulter, Cowell e Jenkins (1992a) e aplica uma abordagem empírica é baseada nas informações da renda e da composição domiciliar brasileiras no ano de 2006. As informações foram extraídas da Pesquisa Nacional por Amostras de Domicílios (PNAD). Os principais resultados evidenciam para uma alteração estatisticamente significativa nos índices, indicando que a consideração de julgamentos morais e/ou parâmetros ad hoc causa feitos relevantes sobre as medidas de desigualdade e de pobreza.
\end{abstract}

Palavras-chave: Desigualdade. Pobreza. Escala equivalente.

Abstract: The objective of this study is to measure the accuracy of some measurements of income inequality and poverty in Brazil taking into account the variations in the equivalent scales and in household composition. The study follows the theoretical reference developed by Coulter, Cowell e Jenkins (1992a) and applies an empirical approach based on the information of income and Brazilian household composition in 2006. The information was gathered in collaboration with the National Household Sample Survey (PNAD). The main results point to a significant statistical alteration in the indexes indicating that

* Os autores gostariam de agradecer ao editor Ricardo Dathein e a um parecerista anônimo. Entretanto, erros e omissões são de nossa inteira responsabilidade. O primeiro autor ressalta a importância do financiamento parcial concedido pelo CNPq, por meio do projeto de número 475225/2009-0.

* * Professor do Programa de Pós-Graduação em Economia da UFPB. Pesquisador do CNPqBrasil. E-mail: eafigueiredo@gmail.com

* * * Professor do Programa de Pós-Graduação em Economia da UFRN. E-mail: jdal@ufrnet.br 
the consideration of moral judgments and/or ad hoc parameters, cause relevant effects in the measurements of inequality and poverty.

Keywords: Inequality. Poverty. Equivalence scale.

JEL Classification: I30; I32; I39.

\section{Introdução}

A literatura relacionada à desigualdade de renda e de pobreza geralmente assume que a renda pessoal é o único atributo relevante para a mensuração dos indicadores. ${ }^{1}$ Tal suposição impossibilita, por exemplo, a comparação do bem-estar dos indivíduos que residem em unidades familiares com diferentes tamanhos e composições e, por conseguinte, com os mais variados níveis de necessidades.

A saída para essa questão é admitir uma escala equivalente. Nesse sentido, a literatura empírica costuma ponderar a renda domiciliar a partir de uma função do tamanho da unidade familiar e de sua composição (por exemplo: número de adultos, de crianças, de mulheres e de idosos). De acordo com Duclos e Araar (2006), esse tipo de ponderação é, normalmente, realizado de forma ad hoc, introduzindo-se, com isso, julgamentos morais e/ou arbitrariedades na análise. ${ }^{2}$ Surge, assim, uma importante questão: qual o impacto dos diferentes julgamentos, ou escalas, sobre as medidas de concentração e de pobreza?

Alguns estudos já destacaram os efeitos das escalas de equivalência sobre as medidas tradicionais de desigualdade e de pobreza. Dentre esses estudos, destacam-se Buhmann et al. (1988) e Coulter, Cowell e Jenkins (1992a; 1992b). Uma aplicação para o Brasil foi realizada por Ferreira, Lanjouw e Neri (2003). Os autores procuraram verificar os efeitos das alterações nos deflatores de preços e escala equivalente sobre os índices de pobreza (proporção de pobres) e de desigualdade de renda (Gini) no Brasil, em 1996. Entre outros resultados, eles perceberam elevações na proporção de pobres e no índice de Gini quando havia mudança na escala equivalente. Em suma, nesses estudos foram observados comportamentos não lineares dos indicadores entre as duas escalas arbitrárias usuais: renda domiciliar e renda per capita. Ou

1 Excluindo-se os estudos que consideram a abordagem das capacitações. Para detalhes, ver Sen (1992).

2 A introdução desses fatores é muito comum nas análises de distribuição de renda e de pobreza. As noções de aversão social à desigualdade e de dominâncias estocásticas de primeira e de segunda ordem como fatores determinantes do nível de bem-estar social servem de exemplo para essa afirmação. Para detalhes, ver, respectivamente, Atkinson (1970) e Shorrocks (1983). 
seja, seus resultados sugerem que os julgamentos sociais relacionados à sensibilidade ao tamanho do domicílio e às diferentes necessidades produzem um efeito relevante no cálculo dos índices.

Com vistas a mensurar esses efeitos, este artigo propõe-se a verificar como as medidas de desigualdade de renda e de pobreza se alteram quando se admitem mudanças nas escalas equivalentes. Para isso, o estudo seguirá o referencial teórico desenvolvido por Coulter, Cowell e Jenkins (1992a) e seguirá uma abordagem empírica baseada nas informações de renda e de composição domiciliar brasileiras no ano de 2006. Os dados serão extraídos da Pesquisa Nacional por Amostras de Domicílios (PNAD).

Este trabalho está organizado em cinco seções, incluindo-se esta introdução. Na segunda seção, apresenta-se a estrutura analítica do artigo (notações e definições utilizadas). A terceira trata do efeito da escala equivalente sobre os índices de desigualdade de renda e de pobreza. Os resultados são apresentados e discutidos na quarta seção e, na última, estão as considerações finais.

\section{Referencial Analítico}

Nesta seção, serão apresentados os principais conceitos utilizados no estudo. Em um primeiro momento, destaca-se o conceito de renda equivalente. Em seguida, apresentam-se alguns indicadores de concentração e de pobreza.

\subsection{Renda e Escala Equivalente}

A unidade de análise deste estudo é a pessoa no domicílio. Admitese que a renda domiciliar é equitativamente distribuída entre os residentes no domicílio, de forma que todas as pessoas recebem rendas equivalentes. O tamanho dos domicílios é representado por: 's', ou seja, o número de pessoas de cada domicílio, $s=1, \ldots, n$. Além disso, representa-se a participação do grupo 's' na população por $p_{s}$, tal que

$$
\sum_{s=1}^{n} p_{s}=1
$$

Assume-se que a renda domiciliar ' $X$ ' é continuamente distribuída no intervalo $[0, a)$. Considera-se, ainda, que domicílios com maior número de pessoas têm grau de necessidades maior do que o dos domicílios menores. O parâmetro $\theta$ é a escala relativa que capta os diferentes graus de necessidades. A escala equivalente para um parâmetro é dada por

$$
M_{S}=M(s, \theta) \text {, em que } \theta \geq 0
$$


sendo $M_{S}$ uma função crescente nos parâmetros $s$ e $\theta$, a renda equivalente, ' $Y_{i}$ ', para cada pessoa, é igual à renda domiciliar dividida pela escala equivalente. Isto é:

$$
Y_{i}=X / M_{S}
$$

A suposição sobre a distribuição de $X$ implica que a renda equivalente também tenha uma distribuição contínua no intervalo $[0, b)$.

A renda média do domicílio no grupo $s$ é $\bar{X}_{S}$; a renda média equivalente no grupo sé $\bar{Y}_{S}=\bar{X}_{S} / M_{S}$; e a renda média da população é $\bar{Y}$. Um caso especial de (1) frequentemente usado é aquele proposto por Buhmann et al. (1988), a saber:

$$
M_{S}=s^{\theta}
$$

Essa formulação simples é usada em estudos empíricos que incluem diversas escalas que são baseadas em outras características dos domicílios, além do tamanho. Os dois casos extremos são representados pela renda per capita, ou seja, $(\theta=1)$ e pela renda domiciliar, quando não se admite nenhum ajustamento $(\theta=0)$.

\subsection{As Medidas de Desigualdade de Renda e de Pobreza}

No sentido de observar a influência da escala equivalente e da composição do domicílio sobre as medidas de desigualdade e de pobreza no Brasil, optou-se por duas famílias de índices. Para mensurar a desigualdade, escolheu-se o índice de entropia generalizada, do qual, com base na escolha da aversão à desigualdade, pode-se derivar, entre outros, o índice de Theil. Para mensurar a pobreza, utilizou-se o índice de Foster-Greer-Thorbecke (FGT), do qual se obtém a proporção de pobres.

\subsection{1 Índice de Entropia Generalizada - EG}

Entre as medidas de desigualdade de renda, considerou-se o índice de entropia generalizada (EG), que é dado pela seguinte fórmula:

$$
I_{\alpha}=[1 / \alpha(\alpha-1)] \int_{0}^{b}\left[(Y / \bar{Y})^{\alpha}-1\right] f(Y) d Y
$$

Observa-se que, para cada membro da medida de desigualdade de Atkinson (1970), há um membro equivalente do EG. De acordo com Couter et al. (1992), quanto maiores os valores dos parâmetros de aversão à desigualdade, $\varepsilon \geq 0$, mais negativos e menores serão os valores do índice de EG $(\alpha=1-\varepsilon)$. Para o caso particular de $\varepsilon=0$, e o valor de $\alpha=1$, obtém-se, assim, o índice de Theil. 


\subsection{2 Índice de Pobreza - FGT}

A família de índices de pobreza estabelecida por Foster, Greer e Thorbecke (1984) e reportada na literatura por FGT é representada por

$$
P_{\beta}=\int_{0}^{z}\left[1-(Y / Z)^{\beta}\right] f(Y) d Y
$$

onde Z é a linha de pobreza, em termos de renda equivalente, portanto, comum a todas as pessoas, sem distinção do tipo de família. Couter et al. (1992, p. 1071) mostram, ainda, que, quanto mais altos os valores de $\beta$, maior será o peso para os grandes hiatos de pobreza, resultando numa maior aversão à pobreza.

Da família de índices de pobreza FGT, para $\beta=0,1$ e 2, obtém-se, respectivamente, a proporção de pobres, $P_{0}$; o hiato de pobreza, $P_{1}$; e o hiato quadrático ou a severidade da pobreza, $P_{2}$.

Assim, usando-se diferentes linhas de pobreza para diferentes tipos de domicílios e levando-se em consideração suas necessidades, tem-se que

$$
Z_{\mathrm{s}}=\mathrm{Z}_{1} \mathrm{M}_{\mathrm{s}}
$$

Em cada grupo, a linha de pobreza é definida como um múltiplo para a única pessoa do domicílio, de modo que o múltiplo é propriamente a taxa de escala equivalente. Coulter, Cowell e Jenkins (1992a) consideram dois casos:

$$
\begin{aligned}
& Z_{1}=\pi \text { (linha de pobreza absoluta) e } \\
& Z_{1}=\lambda \bar{Y} \text { (linha de pobreza relativa), onde os escalares } \pi, \lambda>0 .
\end{aligned}
$$

\subsection{Dados}

Os dados utilizados neste estudo foram captados na Pesquisa Nacional por Amostras de Domicílios (PNAD). A pesquisa, realizada pelo Instituto Brasileiro de Geografia e Estatística (IBGE), desde o final da década de 1960, conta com um questionário básico, envolvendo perguntas relativas a características domiciliares e pessoais, tais como: tamanho da família, renda domiciliar, nível educacional dos moradores, número de horas trabalhadas, renda pessoal, entre outras. Em alguns anos, são investigadas, e sumarizadas em suplementos, algumas características particulares, como: saúde, segurança alimentar, trabalho infantil e mobilidade social.

Além dessas particularidades, a PNAD possui um desenho amostral complexo. Consequentemente, seus dados não são independentes e identicamente distribuídos (i.i.d.), o que pode conduzir a estimativas ineficientes para as medidas de desigualdade e de pobreza (ZHENG, 
2002). Diante disso, o estudo promoveu o ajustamento da amostra, visando a respeitar seu design. O procedimento de ajustamento é apresentado no anexo B.

No que se refere ao cálculo dos indicadores de pobreza e de desigualdade, optou-se por trabalhar com o método de estimação proposto por Kovacevic e Binder (1997). Nesse procedimento, a estimação da variância dos índices é realizada a partir da linearização de Taylor via Estimating Equations (EE). Os autores demonstram que a variância, para o estimador $\hat{\theta}$, será

$$
\operatorname{var}(\hat{\theta})=\operatorname{var}\left(\hat{\theta}-\theta_{N}\right) \approx \operatorname{var}\left(\sum_{s} w_{i} u^{*}\left(y_{i}, \theta_{N}\right)\right),
$$

onde $\theta_{\mathrm{N}}$ representa o verdadeiro parâmetro em uma população finita, $w_{i}$ os pesos associados às observações e

$$
u^{*}\left(y_{i}, \theta_{N}\right)=-J_{\theta}^{-1} u\left(y_{i}, \theta_{N}\right)
$$

com

$$
J_{\theta}=\left.\sum_{i=1}^{N} \frac{\delta u\left(y_{i}, \theta\right)}{\delta \theta}\right|_{\theta=\theta_{N}}
$$

Apresentado o método de estimação, destacam-se as transformações efetuadas sobre a variável de interesse, "renda domiciliar", ${ }^{3}$ a qual foi ajustada ao tamanho do domicilio, obtendo-se a renda equivalente por

$$
Y_{i}=X / s^{\theta} .
$$

A outra transformação leva em conta a composição da família. Nesse caso, utiliza-se a seguinte escala equivalente:

$$
Y_{i}=X /\left[1+\Phi_{1}\left(\mathrm{~N}_{\mathrm{A}}-1\right)+\Phi_{2} \mathrm{~N}_{\mathrm{C}}\right]^{\theta}
$$

onde $\mathrm{N}_{\mathrm{A}}$ e $\mathrm{N}_{\mathrm{C}}$ representam o número de adultos e de crianças, respectivamente, e $\Phi_{1}$ e $\Phi_{2}$ os pesos (entre zero e um) atribuídos ao números de adultos e ao de crianças abaixo de 10 anos de cada domicílio. $O$ peso 1 é atribuído ao chefe do domicílio.

Serão utilizados os seguintes valores para a escala equivalente: $\theta=0,1 ; 0,2 ; \ldots ; 0,9 ; 1$. A composição do domicílio também será levada

3 Diversos trabalhos utilizam essa variável como objeto de análise, podendo-se citar: Jenkins (1995), Burkhauser et al (1999) e Burkhauser et al (2001). É importante destacar que há uma diferença entre renda familiar e renda domiciliar, dado que mais de uma família podem conviver em um mesmo domicílio. No Brasil, cerca de 5\% dos domicílios enquadramse nessa categoria (IPEA, 2006). 
em conta. Nesse caso, será considerado o número de crianças (abaixo de 10 anos) e o de adultos em cada unidade domiciliar. Os valores relativos ao peso das crianças e ao dos adultos seguirão a orientação de Figini (1998). Nesse sentido, tem-se três situações: a) Estrutura I, com $\Phi_{1}$ $=0,7$ e $\Phi_{2}=0,5$; b) Estrutura II, $\operatorname{com} \Phi_{1}=0,6$ e $\Phi_{2}=0,4$ e; c) Estrutura III, com $\Phi_{1}=0,5$ e $\Phi_{2}=0,3$. Nota-se que o peso das crianças é sempre inferior ao dos adultos, assim como considerado em Kakwani (1996).

\section{Mudanças Relativas na Escala Equivalente e os Efeitos sobre as Medidas de Desigualdade e Pobreza}

Segundo Coulter, Cowell e Jenkins (1992a), o efeito de mudanças da escala equivalente sobre as medidas de desigualdade não é imediatamente óbvio, pois depende do impacto do acréscimo de $\theta$ na renda média do grupo e na renda média total. Nesse sentido, os autores mostram que, se a renda domiciliar (renda não ajustada) correlacionar-se positivamente com o tamanho da família, dado um acréscimo em $\theta$, a razão para aqueles domicílios com maior tamanho médio diminui mais do que para os domicílios com menor tamanho médio, resultando num efeito de aproximação da distribuição da renda equivalente. Contudo, essa não é a única influência. Mudanças em $\theta$ podem alterar a classificação dos indivíduos na distribuição da renda equivalente (efeito reclassificação).

3.1 Efeitos da Mudança da Escala Equivalente sobre o Índice de Desigualdade de Entropia Generalizada

Diferenciando-se o índice de entropia em relação a $\theta$, obtém-se

$$
\partial I_{\alpha} / \partial \theta=\bar{Q}_{\alpha s}\left[\operatorname{cov}\left(\bar{Y}_{S}, M_{S}^{\prime}\right) / \bar{Y}\right]-\operatorname{cov}\left(Q_{\alpha s}, M_{S}^{\prime}\right)
$$

$$
\text { onde } \begin{aligned}
& Q_{\alpha s}=\left(\bar{Y}_{s}, \bar{Y}\right)^{\alpha}\left[1+\alpha(\alpha-1) I_{\alpha s}\right] /(\alpha-1), \quad \alpha \neq 1 \\
& Q_{1 s}=\left(\bar{Y}_{s}, \bar{Y}\right)\left[1+\log \left(\bar{Y}_{s}, / \bar{Y}\right)+I_{1 s}\right], \text { para } \alpha=1
\end{aligned}
$$

A contribuição do primeiro termo da equação (6) é dar o gráfico de $\mathrm{I}_{\mathrm{as}}$ contra $\theta$ um formato de $U$ quando $\alpha \leq 1$, e o formato de $\cap$ quando $\alpha \geq 1$.

Em relação ao segundo termo, $\operatorname{cov}\left(Q_{a s}, M_{s}^{\prime}\right)$, é mais difícil estabelecer uma causalidade, pois o resultado da diferenciação de $Q_{a s} \mathrm{em}$ relação ao tamanho do domicílio, $\log (s$,$) dependerá da sensibilidade$ do parâmetro $\alpha$, e do impacto sobre a razão entre a média do grupo e a média total. 
Entretanto há alguns resultados mais evidentes, seja qual for o sinal de $\operatorname{cov}\left(Q_{\alpha s}, M_{S}^{\prime}\right)$. Em relação à curva de Lorenz, para valores baixos de $\theta$, acréscimo no seu valor, promoveria a equalização; isto é,

Em síntese, a previsão mais confiante é que, para valores de $\alpha$ próximos a zero (que não sejam sensíveis nem à base nem ao topo da distribuição), espera-se que o primeiro termo supere o segundo, portanto, o gráfico de $I_{\alpha s}$ contra $\theta$ terá a forma de $U$.

Para medidas sensíveis tanto na base como no topo da distribuição, os resultados não são tão evidentes, pois a $\operatorname{cov}\left(Q_{\alpha s}, M_{S}^{\prime}\right)$ desempenha um papel mais importante. Para medidas sensíveis na base da distribuição, o primeiro termo da equação (6) contribui para um relacionamento na forma de $U$ entre $I_{\alpha}$ e $\theta$. Se, nos valores mais altos de $\theta$ a $\operatorname{cov}\left(Q_{\alpha s}, M_{S}^{\prime}\right)$ for positiva, o gráfico ente $I_{\alpha}$ e $\theta$ terá mais um formato de um $J$ invertido do que um formato de $U$. Se a $\operatorname{cov}\left(Q_{\alpha S}, M_{S}^{\prime}\right)$ for suficientemente negativa para valores altos de $\theta$, o termo da covariância contrabalançará a influência de equalização do primeiro termo.

Para medidas sensíveis ao topo da distribuição, o primeiro termo da equação (6) dará ao gráfico entre $I_{\alpha}$ e $\theta$ com um formato de $U$ invertido. Um gráfico na forma de $\cup$ surgiră se, para valores mais altos de $\theta$, a $\operatorname{cov}\left(Q_{a s}, M_{S}^{\prime}\right)$ for negativa.

Considerando-se que a maioria das medidas de desigualdade mais comumente usadas nos trabalhos empíricos corresponde a membros da família do índice de GE, para $\alpha$ na classe [-1, 2], espera-se, portanto, que seja identificado um relacionamento na forma de $U$ entre $I_{\alpha}$ e $\theta$.

Em relação ao índice de Gini, não se pode analisar de acordo com o raciocínio descrito anteriormente, porque as rendas são agregadas usando-se pesos que dependem da classificação, associadas com cada renda, em vez de o serem com a renda per capita. Entretanto, o relacionamento entre $G$ e $\theta$, provavelmente, terá um formato de $U$ quando mudanças nas classificações da renda (ranks) induzidas por mudanças em $\theta$ forem relativamente pequenas.

\subsection{Efeitos da Mudança da Escala Equivalente sobre Medidas de Pobreza}

Derivando (5) em relação a $\theta$ obtém-se

$$
\partial P_{\beta} / \partial \theta=\sum_{s=1}^{n} p_{s} M_{s}^{\prime} D_{s \beta}\left(1-T_{s}\right)
$$

onde $T_{s} \equiv 0$ (caso da linha de pobreza absoluta);

$$
\equiv\left(1 / M_{s}^{\prime}\right)\left[\operatorname{cov}\left(\bar{Y}_{s}, M_{s}^{\prime}\right) / \bar{Y}+\bar{M}_{s}^{\prime}\right] \text { (caso da linha de pobreza relativa) e }
$$




$$
\begin{aligned}
& D_{\mathrm{s} 0} \equiv \mathrm{g}^{\mathrm{s}}\left(Z_{\mathrm{s}}\right) Z_{\mathrm{s}}>0 \\
& D_{\mathrm{s} \beta} \equiv \beta \int_{0}^{Z_{s}}\left[1-\left(X / Z_{s}\right)\right]^{\beta-1}\left(X / Z_{s}\right) g(X) d X>0, \quad \beta \geq 1
\end{aligned}
$$

O impacto de um acréscimo de $\theta$ sobre essas medidas de pobreza decompõe-se em três efeitos separados. Lembrando-se que $Z_{\mathrm{s}}=Z_{1} \mathrm{M}_{\mathrm{s}}$, percebe-se que um desses efeitos ocorre via mudança em $\mathrm{M}_{\mathrm{s}}$, e os outros dois decorrem das mudanças em $Z_{1}$.

O primeiro efeito é uma linha de pobreza "pura". Quanto mais alta o valor de $\theta$, mais alto será a taxa de escala equivalente para todos os tipos de domicílios e, portanto, mais altas serão as linhas de pobreza. Caso as distribuições de renda não se alterem, a proporção da população pobre, $P_{0}$, deverá aumentar. $O$ segundo efeito reflete mudanças na forma da distribuição da renda abaixo da linha de pobreza. O terceiro efeito do impacto da mudança de $\theta$ sobre os índices de pobreza é um efeito indireto da linha de pobreza, que surge quando se utiliza a linha de pobreza relativa.

$\mathrm{Na}$ análise dos índices de desigualdade e de pobreza, o impacto da mudança relativa na escala equivalente sobre os índices, dever ser observado na mesma direção, seja qual for o valor $\beta$.

\section{Resultados}

Nesta seção, em primeiro lugar, discutir-se-á sobre algumas características preliminares da amostra e, em seguida, dar-se-á atenção aos resultados relativos à sensibilidade das medidas de desigualdade e de pobreza, frente à sensibilidade relativa ao tamanho e a sua composição da família.

\subsection{Análise Preliminar}

A Tabela 1 sumariza as principais informações estatísticas da amostra. Em primeiro lugar, observa-se que a maioria dos domicílios brasileiros (cerca de 78\%) são compostos por até quatro pessoas. Da terceira coluna em diante, tem-se a participação da renda média de cada estrutura domiciliar, de acordo com os diversos valores de $\theta$. Nota-se que, à exceção do último estrato (domicílios com seis ou mais membros), a proporção da renda equivalente cresce à medida que cresce o tamanho do domicílio. Esse padrão é observado para pequenos valores da sensibilidade (especificamente, $\theta=0$ e $\theta=0,25$ ). A partir do $\theta=0,50$, a lógica inverte-se. Ou seja: os domicílios menores tendem a apresentar maior renda equivalente. 
Tabela 1 - Média da renda equivalente por tamanho do domicílio e $\theta$

\begin{tabular}{|c|c|c|c|c|c|c|}
\hline \multirow{3}{*}{$\begin{array}{l}\text { Tamanho do } \\
\text { domicilio }\end{array}$} & \multirow{3}{*}{$\begin{array}{l}\text { Tamanho do } \\
\text { domicílio (\%) }\end{array}$} & \multirow{2}{*}{\multicolumn{5}{|c|}{$\begin{array}{l}\text { Média da renda equivalente (como um \% da média total) } \\
100 \times\left(\bar{Y}_{s} / \bar{Y}\right)\end{array}$}} \\
\hline & & & & & & \\
\hline & & $\theta=0,00$ & $\theta=0,25$ & $\theta=0,50$ & $\theta=0,75$ & $\theta=1,00$ \\
\hline 1 & 11 & 67 & 84 & 116 & 142 & 188 \\
\hline 2 & 20 & 96 & 105 & 118 & 126 & 135 \\
\hline 3 & 24 & 101 & 102 & 101 & 99 & 94 \\
\hline 4 & 23 & 115 & 109 & 100 & 92 & 81 \\
\hline 5 & 12 & 109 & 116 & 84 & 75 & 61 \\
\hline $6+$ & 10 & 97 & 83 & 65 & 54 & 41 \\
\hline $\begin{array}{l}\text { Renda Média } \\
\text { Total }\end{array}$ & & 1.623 & 1.292 & 935 & 764 & 577 \\
\hline $\operatorname{cov}[Y, \log (s)] / \bar{Y}$ & & 0,054 & 0,004 & $-0,075$ & $-0,133$ & $-0,223$ \\
\hline
\end{tabular}

Fonte: Dados da pesquisa.

Outro comportamento de destaque é que a renda dos domicílios pequenos (com até dois componentes) cresce à medida que $\theta$ cresce. Já nos domicílios maiores (com mais de três componentes), o comportamento é inverso. Esse padrão é refletido pela covariância normalizada entre a renda ajustada e o logaritmo do tamanho do domicílio, o qual se torna negativa a partir do $\theta=0,50$.

As subseções seguintes serão destinadas à apresentação dos resultados. Em primeiro lugar, será destacado o comportamento das medidas de desigualdade e de pobreza frente à variação no parâmetro de sensibilidade e, em seguida, serão investigados os efeitos das variações no parâmetro de sensibilidade e na composição do domicílio.

\subsection{Efeitos da Variação da Escala Equivalente nas Medidas de Desigualdade}

A análise empírica atende a diversos propósitos. Em primeiro lugar, serve para comprovação dos resultados teóricos desenvolvidos nas seções 2 e 3. Em segundo, acredita-se que as sensibilidades dos indicadores de concentração de renda e das medidas de pobreza devem ser consideradas em uma estratégia política voltada para a distribuição de renda.

Sob o ponto de vista teórico, espera-se que as principais medidas de concentração de renda apresentem um formato de U. Ou seja, iniciando-se com uma sensibilidade igual a zero, o crescimento de $\theta$ tende a equalizar a curva de Lorenz, reduzindo o nível de desigualdade. Porém, a partir de um determinado valor de $\theta$, a medida de desigual- 
dade torna a crescer.

A observação dos índices de Gini e Theil, (Figuras 1 e 2) parece confirmar esse comportamento teórico. Contudo, um olhar mais cuidadoso indica que as curvas estão mais próximas de um $\mathrm{J}$ que de um $\mathrm{U}$. Ou seja, a curva de Lorenz distancia-se da linha de igualdade quando a sensibilidade se aproxima de 1 . Vale salientar que esse comportamento também é observado nos índices de Atkinson (1970) e na variância do logaritmo, (Figuras A.1, A.2 e A.3 em anexo). Uma explicação possível é esta: quando $\theta \rightarrow 1$, há uma redução no share da renda dos mais pobres, seguida pelo aumento na proporção da renda dos mais ricos. Com isso, o índice de desigualdade cresce. É importante salientar que as menores rendas equivalentes, entre as famílias com mais de um membro, são observadas entre os domicílios com seis ou mais membros (Tabela 1).

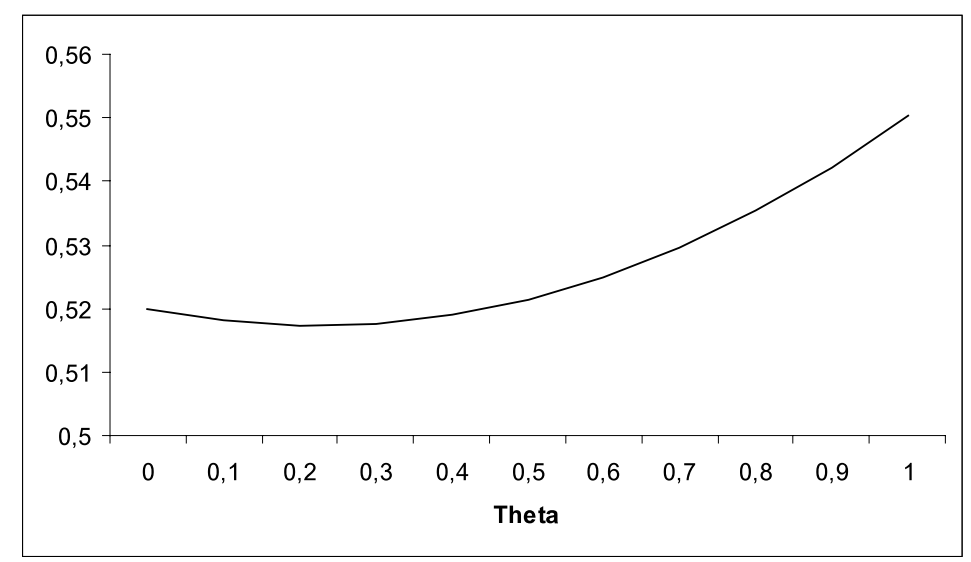

Figura 1 - Índice de Gini

Fonte: PNAD (2006).

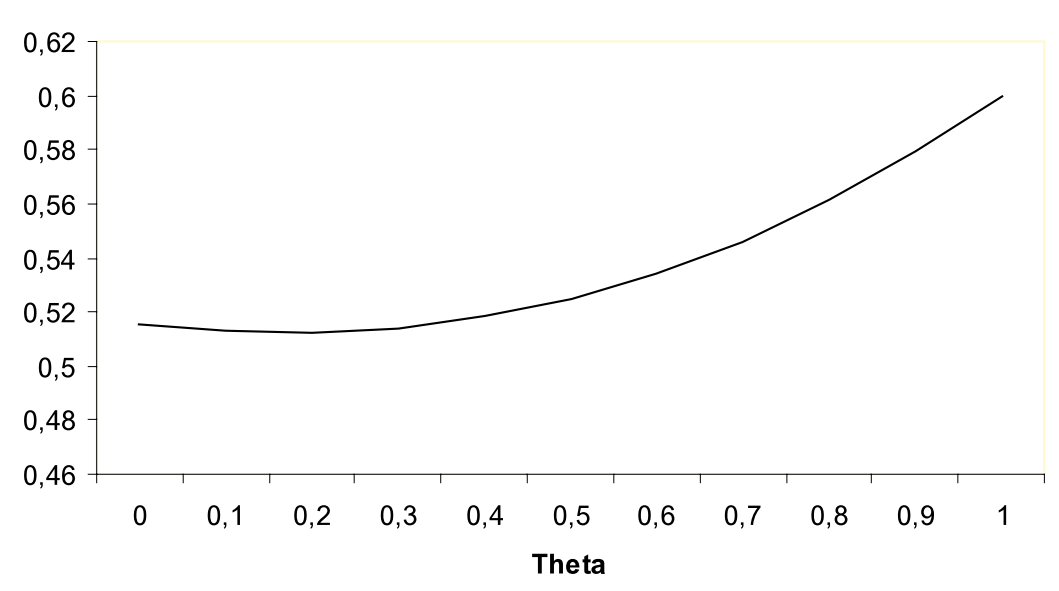

Figura 2 - Índice de Theil

Fonte: PNAD (2006).

Grosso modo, observou-se uma variação de $6 \%$ no índice de Gini, e de $16 \%$ no Theil, quando transitou-se da renda domiciliar para a renda 
per capita. Essas variações podem ser consideradas expressivas e estavam próximas às mudanças observadas por Coulter, Cowell e Jenkins (1992a) ao investigarem dados do Reino Unido. Entretanto, elas são estatisticamente significativas? Para responder a essa questão foram calculados os intervalos de confiança dos indicadores, considerandose um desvio padrão mensurado via linearização de Taylor, seguindo a orientação de Kovaevic e Binder (1997). Os resultados das estimações estão sumarizados na Tabela 2.

Tabela 2 - Indicadores de Desigualdade (valores selecionados de $\theta$ )

\begin{tabular}{lll}
\hline \multirow{2}{*}{ Sensibilidade } & Indicadores & Theil \\
\cline { 2 - 3 }$\theta=0,00$ & Gini & 0,5152 \\
& 0,5199 & {$[0,5058 ; 0,5253]$} \\
$\theta=0,50$ & {$[0,5149 ; 0,5250]$} & 0,5249 \\
& 0,5214 & {$[0,5177 ; 0,5243]$} \\
$\theta=1,00$ & {$[0,5159 ; 0,5268]$} & 0,6001 \\
& 0,5502 & {$[0,5904 ; 0,6122]$} \\
\hline
\end{tabular}

Fonte: Dados da pesquisa.

Nota: Intervalo com $95 \%$ de confiança entre colchetes.

Nota-se que a variação nos indicadores de desigualdade foi estatisticamente significativa para ambas as medidas, quando comparadas a renda domiciliar e a per capita. De uma forma prática, na transição de uma situação extrema para outra, os valores dos índices se alteraram fora dos seus intervalos de confiança.

\subsection{Efeitos da Variação da Escala Equivalente nas Medidas de Pobreza (FGT)}

No que se refere às medidas de pobreza observaram-se comportamentos similares ao das medidas de desigualdade. Em suma, foram considerados apenas os indicadores de proporção de pobres e de severidade da pobreza (FGT, ${ }^{4} \mathrm{com}$ um $\beta=2$ ). As linhas de pobreza variaram de acordo com o parâmetro de sensibilidade (adotou-se um limiar de um meio do valor mediano). Os resultados contidos nas Figuras 3 e 4 indicam um comportamento próximo a J, assim como observado nos índices de desigualdade.

4 Uma ampla discussão relacionada aos conceitos e às medidas de pobreza pode ser encontrada em Duclos e Araar (2006). 


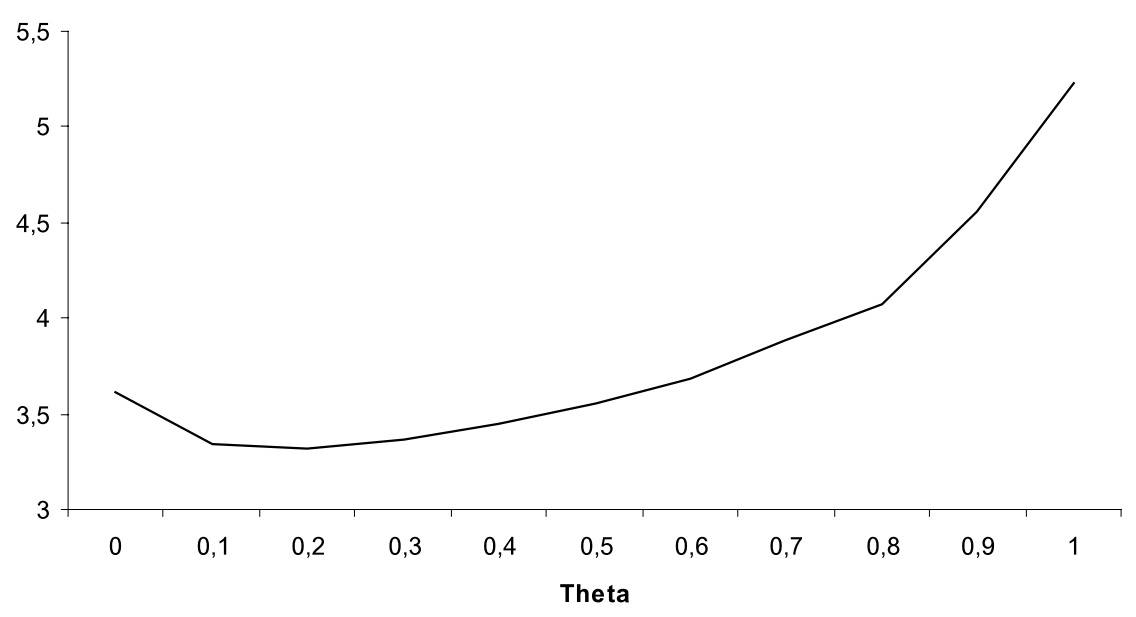

Figura 3 - Medida de Pobreza - FGT Fonte: PNAD (2006).

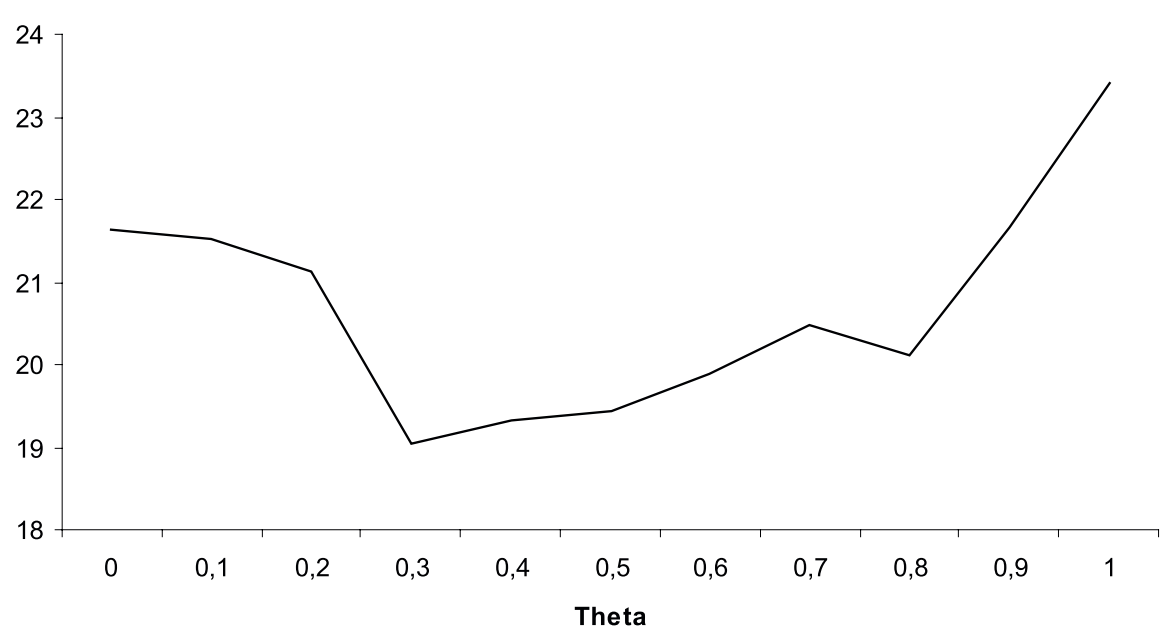

Figura 4 - Medida de Pobreza - Proporção de Pobres Fonte: PNAD (2006).

Os dados indicam que, na transição entre a renda domiciliar e a per capita, houve um aumento de 44\% no FGT e de $8 \%$ na proporção de pobres. Novamente, o cálculo dos intervalos de confiança contido na Tabela 3 aponta para a significância estatística das alterações.

Tabela 3 - Indicadores de Pobreza (valores selecionados de $\theta$ )

\begin{tabular}{llll}
\hline & Indicadores & \\
\cline { 2 - 4 } Sensibilidade & FGT & Proporção & \\
\hline$\theta=0,00$ & 0,0378 & 0,2286 & \\
& {$[0,0367 ; 0,0382]$} & {$[0,2251 ; 0,2313]$} \\
$\theta=0,50$ & 0,0358 & 0,1943 & \\
\hline & & & (continua...)
\end{tabular}




\begin{tabular}{lll}
\hline$\theta=1,00$ & {$[0,0352 ; 0,0360]$} & {$[0,1917 ; 0,1960]$} \\
& 0,0522 & 0,2340 \\
\hline & {$[0,0505 ; 0,0540]$} & {$[0,2305 ; 0,2369]$} \\
\hline
\end{tabular}

Fonte: Dados da pesquisa.

Nota: Intervalo com $95 \%$ de confiança entre colchetes.

Os resultados relativos às medidas de concentração e de pobreza dessa subseção reforçaram as conclusões extraídas do comportamento das medidas de desigualdade. Ou seja: as medidas de pobreza também sofrem um impacto significativo quando se alteram as escalas equivalentes. Contudo, contrariando a expectativa teórica, as curvas assemelham-se mais a uma forma $\mathrm{J}$ do que a um $\mathrm{U}$, isto é, as medidas apresentam maior sensibilidade no topo da escala.

A comparação dos comportamentos dos indicadores de desigualdade e pobreza brasileiros com os internacionais salienta algumas diferenças, mais especificamente em relação aos estudos de Coulter, Cowell e Jenkins (1992a), utilizando os dados do Reino Unido relativos ao ano de 1986 (UK Family Expenditure Survey), e de Figini (1998), investigando o comportamento dos países que compõem o Luxembourg Income Study. A diferença marcante diz respeito ao comportamento da desigualdade e da pobreza quando o $\theta$ se aproxima de 1 . Nesses estudos, os indicadores apresentam um formato de $\mathrm{U}$, porém com valores maiores quando o se aproxima de zero. No caso brasileiro, essa lógica inverte-se, isto é, os valores do índice são maiores quando o $\theta$ se aproxima de 1 e a curva se assemelha mais a um J. Ou seja, as medidas apresentam uma maior sensibilidade no topo da escala.

Em suma, constatou-se que o parâmetro de sensibilidade ao tamanho da família produz mudanças significativas nas medidas de desigualdade e de pobreza. Nesse sentido, surge outra questão: a composição da família também interfere no cálculo desses índices? A subseção seguinte buscará responder a esse questionamento.

\subsection{Efeitos da Variação na Escala Equivalente e na Composição da Familia}

A análise da composição do domicílio será pautada em três situações: a) Estrutura I, com $\Phi 1=0,7$ e $\Phi 2=0,5$; b) Estrutura II, com $\Phi 1=0,6$ e $\Phi 2=0,4$ e; c) Estrutura III, com $\Phi 1=0,5$ e $\Phi 2=0,3$. As Tabelas 4 e 5 apresentam os resultados dos índices de Gini e de Theil para as Estruturas I, II e III e alguns parâmetros de sensibilidade selecionados. Regra 
geral: não há uma mudança significativa entre as estruturas. Contudo, observam-se movimentos relevantes para os diferentes parâmetros de sensibilidade, reforçando os resultados das subseções anteriores.

Tabela 4 - Indicador de Desigualdade de Gini (Diferentes Composições do Domicílio)

\begin{tabular}{llll}
\hline \multirow{2}{*}{ Sensibilidade } & Gini & & \\
\cline { 2 - 4 } & Estrutura I & Estrutura II & Estrutura III \\
\hline$\theta=0,10$ & 0,5181 & 0,5182 & 0,5184 \\
& {$[0,5158 ; 0,5213]$} & {$[0,5149 ; 0,5207]$} & {$[0,5157 ; 0,5217]$} \\
\hline \multirow{2}{*}{$\theta=0,50$} & 0,5177 & 0,5170 & 0,5164 \\
& {$[0,5140 ; 0,5201]$} & {$[0,5145 ; 0,5202]$} & {$[0,5130 ; 0,5189]$} \\
$\theta=1,00$ & 0,5335 & 0,5293 & 0,5250 \\
\hline
\end{tabular}

Fonte: Dados da pesquisa.

Nota: Intervalo com $95 \%$ de confiança entre colchetes.

Tabela 5 - Indicador de Desigualdade de Theil (Diferentes Composições do Domicílio)

\begin{tabular}{llll}
\hline \multirow{2}{*}{ Sensibilidade } & Theil & & \\
\cline { 2 - 4 } & Estrutura I & Estrutura II & Estrutura III \\
\hline$\theta=0,10$ & 0,5124 & 0,5125 & 0,5126 \\
& {$[0,5159 ; 0,5211]$} & {$[0,5056 ; 0,5199]$} & {$[0,5067 ; 0,5210]$} \\
$\theta=0,50$ & 0,5161 & 0,5142 & 0,5124 \\
& {$[0,5042 ; 0,5221]$} & {$[0,5068 ; 0,5212]$} & {$[0,5045 ; 0,5200]$} \\
$\theta=1,00$ & 0,5577 & 0,5468 & 0,5360 \\
& {$[0,5478 ; 0,5679]$} & {$[0,5400 ; 0,5561]$} & {$[0,5271 ; 0,5433]$} \\
\hline
\end{tabular}

Fonte: Dados da pesquisa.

Nota: Intervalo com $95 \%$ de confiança entre colchetes.

Quando comparam-se os resultados obtidos com e sem a consideração da composição do domicílio, observam-se reduções nos índices de desigualdade. Por exemplo: para um $\theta=0,50$, o índice de Gini sem a composição do domicílio, é de 0,5214 (Tabela 2); quando se consideram diferentes pesos dos componentes do domicílio, ele reduz-se para 0,5177 (Tabela 4, Estrutura I). 


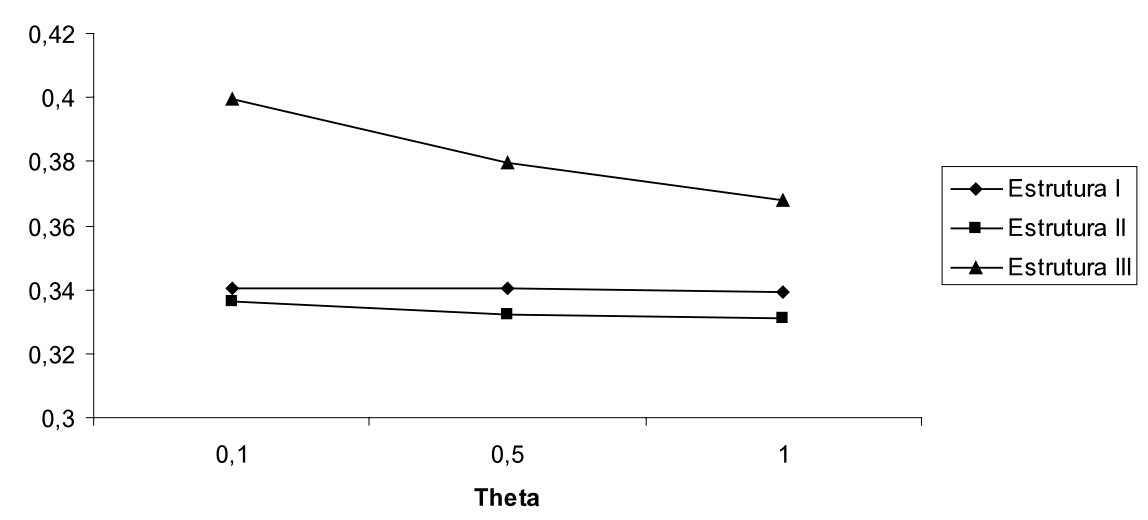

Figura 5 - Medida de Pobreza - FGT (composição do domicílio) Fonte: PNAD (2006).

O comportamento dos índices de pobreza (FGT) é sintetizado na Figura 4.5. De uma forma geral, controlados pela composição do domicílio, os índices mantêm uma relação inversa com o parâmetro de sensibilidade ao tamanho do domicílio $(\theta)$. Novamente, a composição parece contrabalançar o efeito da sensibilidade. Observa-se também que a Estrutura III está exposta aos maiores índices. Ou seja, quanto menor o peso dos indivíduos dentro do domicílio, maior a pobreza. De acordo com Figini (1998), esse comportamento deve-se à distribuição dos adultos e das crianças dentro dos domicílios. Normalmente, o número de adultos dentro do domicílio cresce ao longo da distribuição. Assim, fixado o peso das crianças, um acréscimo no peso dos adultos reduz a desigualdade. Contudo, assim como nos índices de desigualdade, essa mudança não foi estatisticamente significativa.

\section{Considerações Finais}

Este artigo mensurou o impacto das escalas equivalentes sobre os indicadores de desigualdade e de pobreza. Para isso, duas estratégias foram adotadas: a primeira consistiu em variar-se o parâmetro de sensibilidade da renda em relação ao tamanho do domićlio; na segunda, foram incorporados os efeitos da composição do domicílio.

De uma forma geral, observou-se que a fixação arbitrária de escalas interfere de maneira estatisticamente significativa nos valores dos índices. A consideração do parâmetro de sensibilidade fez com que os indicadores de desigualdade (Gini e Theil) apresentassem um formato de J. Ou seja, as medidas mostraram maior sensibilidade no topo da escala. Os indicadores de pobreza comportaram-se de maneira similar.

Quando adotou-se a sensibilidade à composição do domicílio (nú- 
mero de adultos e de crianças), observou-se uma relação inversa entre os indicadores (pobreza e desigualdade) e o parâmetro de sensibilidade. Ou seja, quando controlada a composição do domicílio, os índices tendem a reduzir quando o $\theta \rightarrow 1$.

Entretanto, esses resultados lançam algumas dúvidas quanto ao processo de mensuração do bem-estar via indicadores de concentração de renda e de pobreza, tais como: qual seria o nível de escala equivalente adequado aos dados brasileiros? Quais os pesos que devem ser atribuídos para a composição do domicílio? Estas questões podem servir como ponto de partida para o desenvolvimento de pesquisas futuras.

\section{Referências}

ATKINSON, A. On the measurement of inequality. Journal of Economic Theory, v. 2, n. 3, p. 244-263, sep. 1970.

BUHMANN, A. B et al.. Equivalence scales, well-being, inequality, and poverty: sensitivity estimates across ten countries using the Luxembourg Income Study (LIS) database. Review of Income $\mathcal{E}$ Wealth, v. 34, n. 2, p. 115-142, jun. 1988.

BURKHAUSER, R. V. et al.. Testing the significance of income distribution changes over the 1980s business cycle: a cross-national comparison. Journal of Applied Econometrics, v. 14, n. 3, p. 253-272, may-jun. 1999.

BURKHAUSER, R. V.; LILLARD, D.; VALENTI, P. How exits from the labor force or death impact household income: a four country comparison of public and private income support. BHPS Conference Essex-UK, 2001.

COULTER, F. A. E.; COWELL, F. A; JENKINS, S. P. Equivalence scale relativities and the extent of inequality and poverty. The Economic Journal, v. 102, n. 414, p. 1067-1082, sep. 1992a.

. Differences in needs and assessment of income distributions. Bulletin of Economic

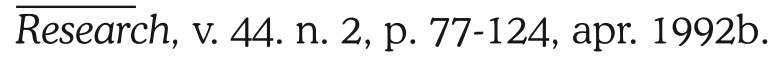

DUCLOS, J-Y.; ARAAR, A. Poverty and equity: measurement, policy, and estimation with DAD. New York, Springer 2006.

FERREIRA, F. G. H.; LANJOUW, P.; NERI, M. A. Robust poverty profile for Brazil using multiple data sources. Revista Brasileira de Economia, Rio de Janeiro, v. 57, n. 1, p. 5992, jan./mar. 2003.

FIGINI, P. Inequality measures, equivalence scales and adjustment for household size and composition. Working Paper, n. 185. Department of Economic, Trinity College, jun. 1998.

FOSTER, J.; GREER, J.; THORBECKE, E. A class of decomposable poverty measures. Econometrica, v. 52, n. 3, p. 761-766, may 1984.

INSTITUTO DE PESQUISA ECONÔMICA APLICADA - IPEA. Sobre a recente queda na desigualdade de renda no Brasil. Brasília: IPEA, 2006. (Nota Técnica).

JENKINS, S. P. Did the middle class shrink during the 1980s? UK evidence from kernel density estimates. Economics Letters, v. 49, n. 4, p. 407-413, oct. 1995. 
. Estimation and interpretation of measures of inequality, poverty, and social welfare using Stata. Stata Users Group, North American Stata Users' Group Meetings 2006. Disponível em: <http://ideas.repec.org/p/boc/asug06/16.html > . Acesso em 30 jul. 2009.

KAKWANI, N. Economic growth, poverty and government. income suport programmes with applications to Australia. In: BALDASSARI et al.. Equity, efficiency and growth: the future of Welfare State. London: MacMillan, 1996.

KOVACEVIC, M.; BINDER, D. Variance estimation for measures of income inequality and polarization. Journal of Official Statistics, v. 13, n. 1, p. 41-58, 1997.

LIMA, J. Aula prática "análise de dados amostrais complexos usando o STATA". Universidade Federal de Viçosa, 2008.

SEN, A. Inequality reexamined. Cambridge: Havard University Press, 1992.

SHORROCKS, A. Ranking income distributions. Economica, v. 50, n. 197, p. 3-17, 1983.

ZHENG, B. Testing Lorenz curves with non-simple random samples. Econometrica, v. 70, n. 3, p. 1235-1243, 2002. 
Anexo A

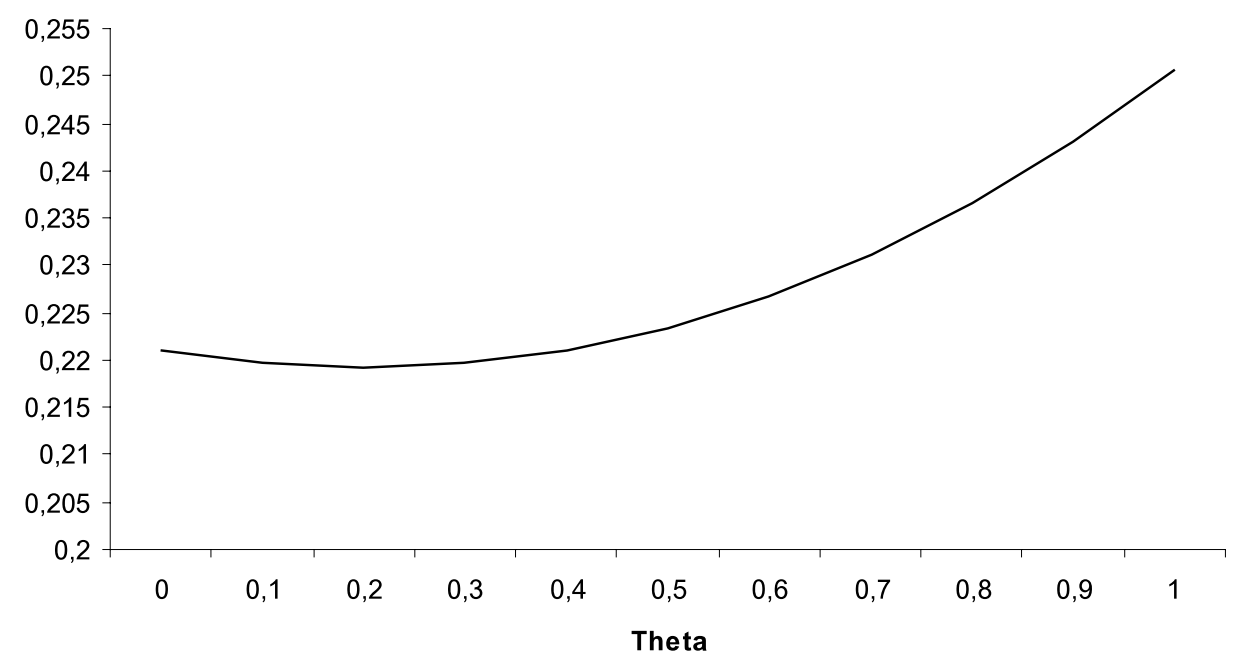

Figura A 1 - Índice de Atkinson (parâmetro de aversão à desigualdade igual a 0,5$)$

Fonte: PNAD (2006).

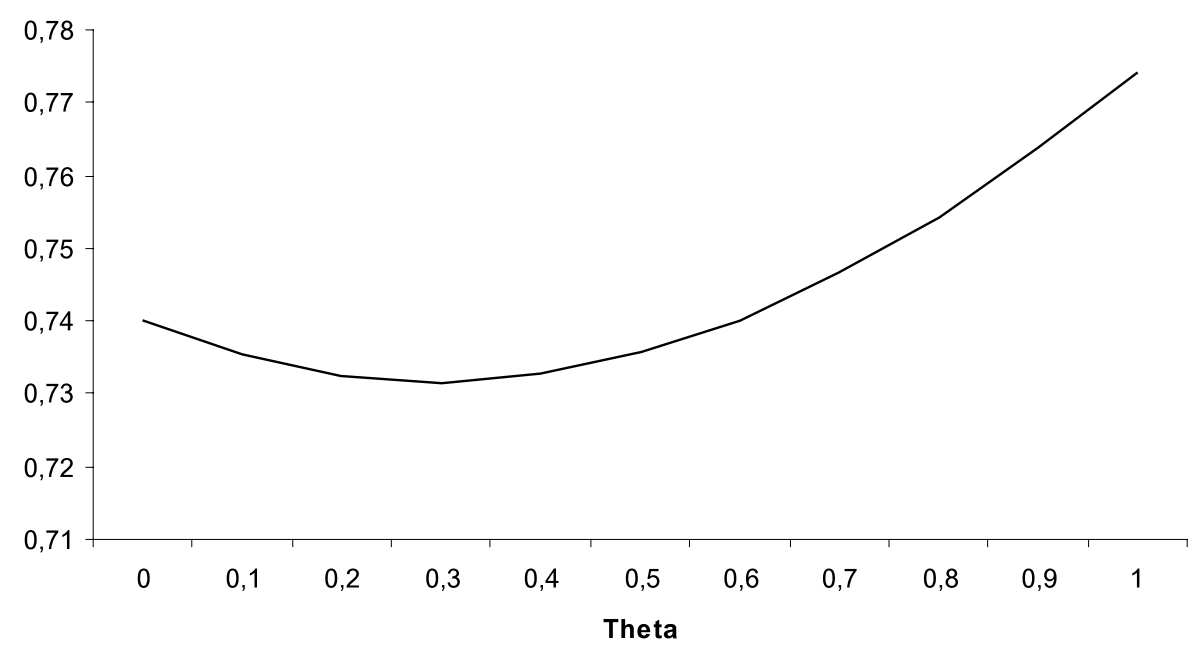

Figura A 2 - Índice de Atkinson (parâmetro de aversão à desigualdade igual a 2,5)

Fonte: PNAD (2006). 


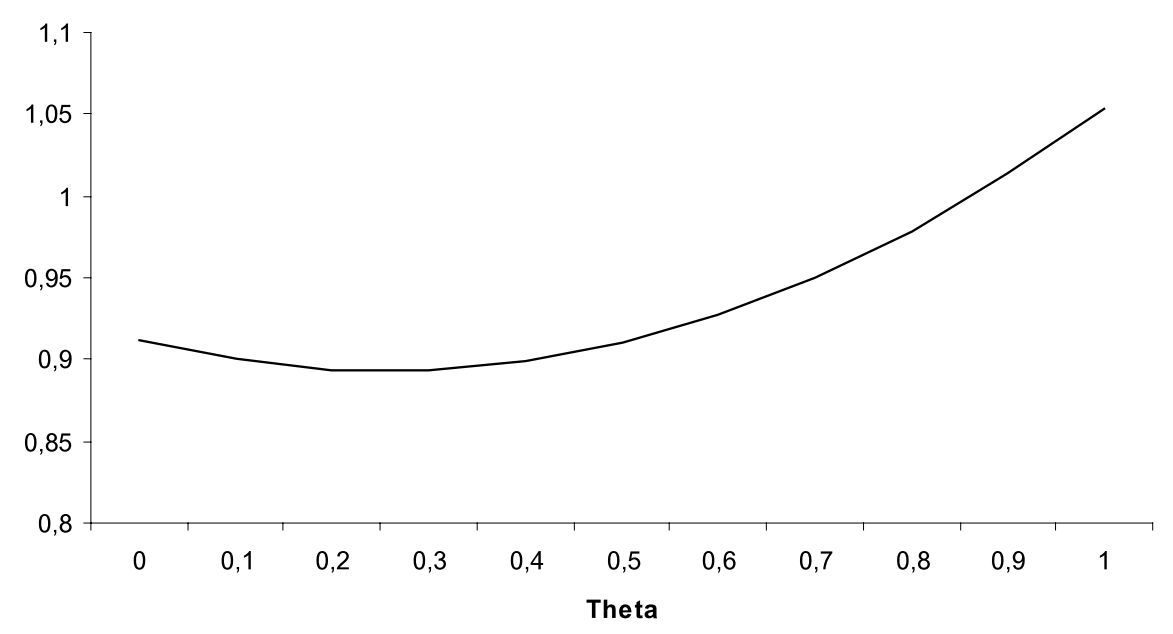

Figura A 3 - Variância do logaritmo

Fonte: PNAD (2006).

\section{Anexo B}

Todos os procedimentos empíricos foram realizados no software STATA 10. Em um primeiro momento, efetuou-se a captação e o ajustamento da amostra a partir de seu desenho complexo. O "do file" utilizado nesta etapa se baseia em Lima (2008) e é apresentado a seguir: ${ }^{5}$

$* * * * * * * * * * *$ Captação dos dados e complexidade amostral

clear

cap log close

log using sensibilidade, replace

set more off

set memory $256 \mathrm{~m}$

infix nt 18-19 nc 20-21 peso 141-145 ano 1-4 uf 5-6

//I

controle 5-12 serie 13-15 tipo 16-17 renda 146-157

//I

espdom 22-22 strat 161-167 psu 168-174 sitcen 83-83

//I

using CDDOMC.txt

\#delimit;

sort controle serie, stable;

5 Seguindo a orientação de um parecerista anônimo, algumas modificações foram efetuadas visando a corrigir o problema da realocação das unidades primarias amostrais (UPA). 
format controle $\% 15.0 \mathrm{~g}$;

format serie \%15.0g;

replace controle $=$ float(controle);

replace serie $=$ float(serie);

keep if espdom $==1$;

\#delimit cr

save desigual06, replace

********Homogeneização dos dados

drop if renda $==0 \mid$ renda $==$.

gen $r=$ renda

drop if $r>30000$

******** Criação dos dados

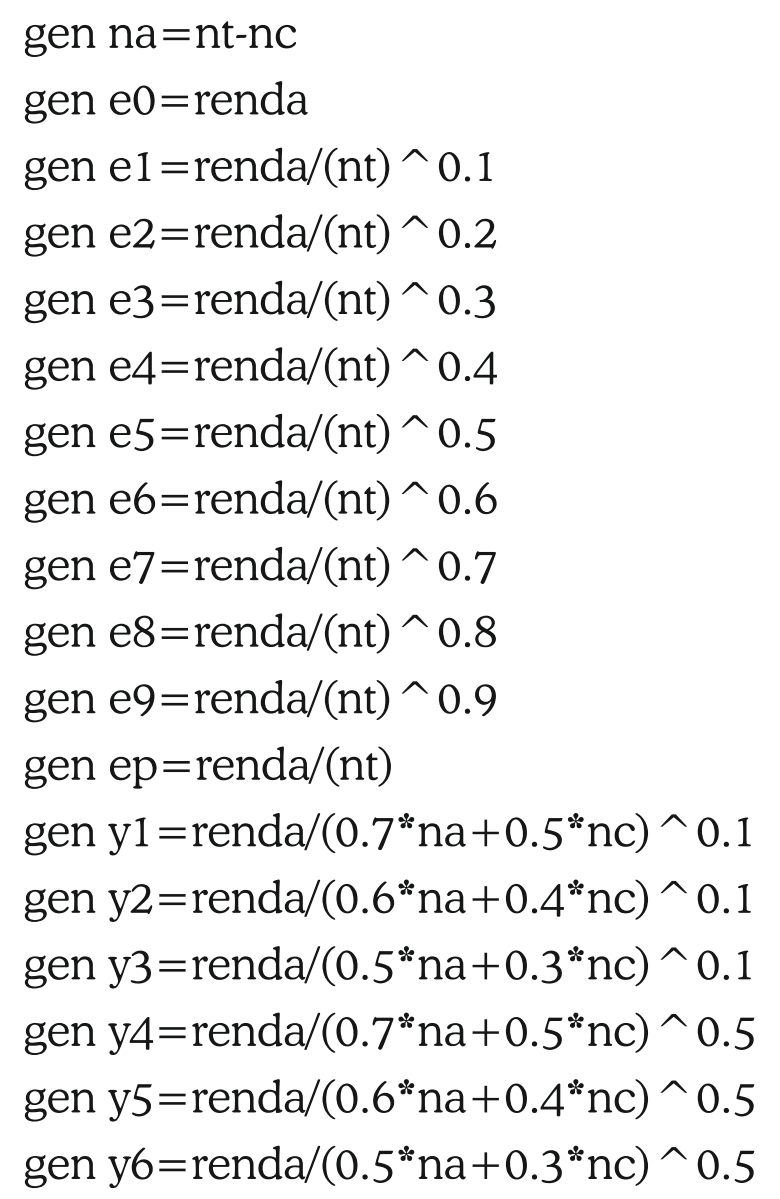


gen $\mathrm{y} 7=\mathrm{renda} /\left(0.7^{*} \mathrm{na}+0.5^{*} \mathrm{nc}\right)$

gen $\mathrm{y} 8=\mathrm{renda} /\left(0.6 * \mathrm{na}+0.4^{*} \mathrm{nc}\right)$

gen $\mathrm{y} 9=\mathrm{renda} /\left(0.5^{*} \mathrm{na}+0.3^{*} \mathrm{nc}\right)$

save desigual06, replace

******Procedimentos relativos a amostra complexa

svyset psu [pweight=peso], strata(strat) vce(linearized) singleunit(missing) svydes, single

* ROTINA DE ALOCAÇÃO DE ESTRATOS COM UM ÚNICO PSU EM ESTRATOS COM MAIOR NUMERO DE OBSERVAÇÕES UTILIZANDO O DO.FILE idonepsu - ANO DE 2006

use desigual06, clear

keep if uf $<11$

gene novo_str $=$.

gene novo_psu $=$.

format novo_psu \% $12.0 \mathrm{~g}$

save acum, replace

cap prog drop prog1

progr define prog 1

use desigual06, clear

keep if uf $==$ estado

gene novo_str $=$ strat

gene novo_psu $=$ psu

qui \{

save transf, replace

use acum, replace

append using transf

save acum, replace

\}

end 


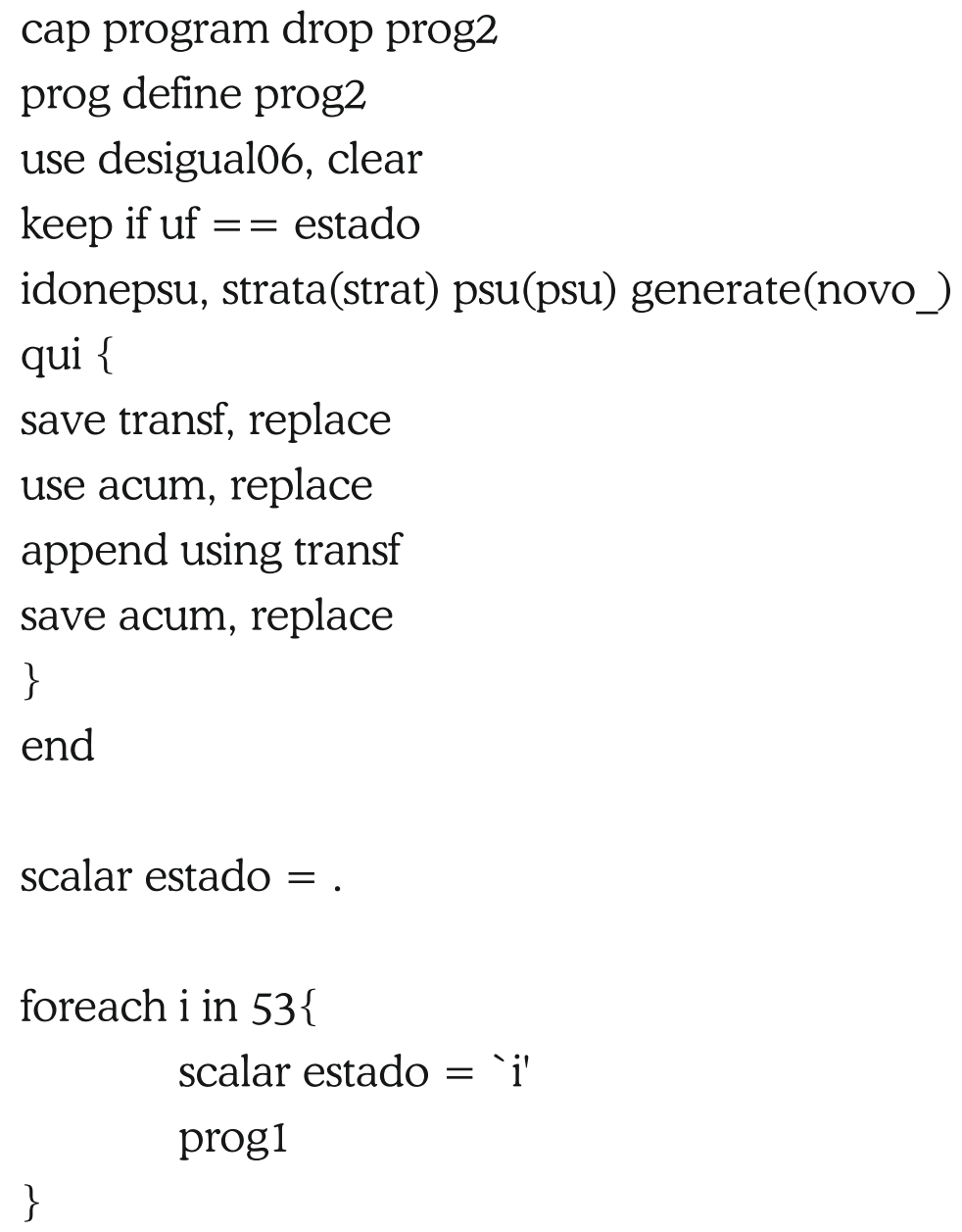


O cálculo dos indicadores foi efetuado a partir dos comandos SVY do STATA. Eles são sumarizados em Jenkins (2006). Por exemplo, a estimativa do índice de Gini com variância obtida a partir do método de linearização de Taylor é obtida a partir de: svylorenz.

Recebido: 27/10/2009.

Aceito: 30/04/2010. 